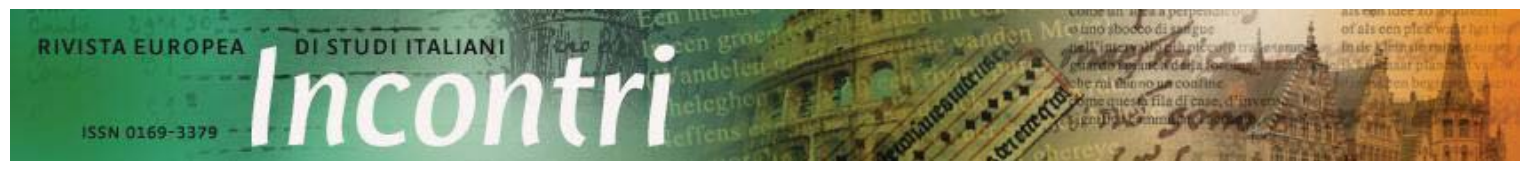

URN:NBN:NL:Ul:10-1-115742 - Publisher: Igitur publishing

Content is licensed under a Creative Commons Attribution 3.0 License

Anno 28, 2013 / Fascicolo 2 - Website: www.rivista-incontri.nl

\title{
La parte maledetta: ciò che è vivo e ciò che è morto nel pensiero italiano del dopoguerra
}

\author{
Recensione di: Dario Gentili, Italian Theory. Dall'operaismo alla \\ biopolitica, Bologna, il Mulino, 2012, 246 p., ISBN: 9788815240262, € \\ 20,00 .
}

Federico Luisetti

Come il suo titolo, sospeso tra uno sguardo da lontano sull'Europa e le vicende italiane, Italian Theory. Dall'operaismo alla biopolitica (il Mulino, 2012) è un racconto a due facce, continuo ma senza scioglimento, un volume che si apre con sinisteritas - "parola latina che indica la "parte maledetta", che induce all" "errore" $\mathrm{e}$, in generale, a "errare" e "deviare" rispetto alla linea e alla via "retta"' (12) - e si conclude con una lunga sequenza di interrogativi sul rapporto tra politica e vita, conflitto e immanenza, globalizzazione e antagonismo.

Senza risolvere la felice tensione produttiva tra esposizione didattica e interpretazione saggistica, Dario Gentili - filosofo della politica già autore di monografie su Walter Benjamin e la topografia politica - tesse una narrazione del pensiero radicale italiano dal secondo dopoguerra ad oggi, privilegiato non per il suo valore accademico ma per la sua singolare accettazione delle contraddizioni, e in seguito del collasso, della tradizione politica occidentale. A differenza dei superamenti o esaurimenti dialettici della modernità euro-atlantica, tipici delle filosofie del postmoderno, secondo Gentili l'operaismo, il pensiero della differenza femminile, le filosofie della crisi e dell'impolitico e, a partire dagli anni Novanta, le varie declinazioni italiane della biopolitica foucaultiana, hanno avuto infatti il coraggio di ingaggiare un corpo a corpo con le categorie politiche della modernità, 'scavalcandole, decostruendole e dislocandole' (9) alla ricerca dei loro nuclei più eversivi.

Per un protagonista del primo operaismo quale Mario Tronti, questa sfida si arresta negli anni Settanta con la diagnosi del definitivo tramonto dell'Occidente e del 'grande Novecento', con un congedo dal vocabolario della lotta di classe e della cultura borghese. Nell'operaismo di Antonio Negri, nel pensiero della crisi di Massimo Cacciari e nella biopolitica di Roberto Esposito e Giorgio Agamben, la posta in gioco è invece il ripensamento del Politico, la collocazione dell'azione all'interno della vita, il tentativo di articolare azione politica e conflitto nell'ambito ormai planetario dei meccanismi di controllo del capitalismo, del suo dominio sui viventi. 
Gentili innesta il proprio discorso in un dibattito ormai consolidato, soprattutto in ambito anglo-americano, sull' 'Italian Theory', ossia sugli aspetti originali del pensiero italiano più radicale e meno accademico, sulla sua differenza nei confronti delle tradizioni filosofiche continentali. Negri ed Esposito hanno contribuito di recente a questa discussione, indicando in una prossimità alla vita, alla storia e alla politica le caratteristiche centrali della riflessione italiana (cfr. Antonio Negri, La differenza italiana, Nottetempo, 2004 e Roberto Esposito, Pensiero vivente. Origine e attulità della filosofia italiana, Einaudi, 2010). Gentili prosegue questa tendenza, sottolineando l'esito biopolitico del pensiero radicale italiano e smarcandosi sia dall'ortodossia operaista di Negri che dal recupero di Esposito della linea umanistica, storicistica e gramsciana.

Procedendo per blocchi cronologici, Gentili scrive un racconto critico-decostruttivo (non tanto storia del pensiero ma cronaca demistificante del potere-sapere), del congedo dal marxismo togliattiano, dalla tradizione storicista, e dalla filosofia della prassi gramsciana. Una storia incentrata sull'esodo a sinistra dalla linea culturale del Partito Comunista Italiano, che si apre non a caso sulla scena gramsciana del marxismo togliattiano e prosegue con il ritorno eretico a Marx - anti-hegeliano e anti-storicistico di Galvano della Volpe e di Tronti, il cui Operai e capitale (1966) è indicato come insuperabile libro-evento del pensiero radicale italiano del dopoguerra.

'Italian Theory' scorge in questa 'parte maledetta', nel discorso settario degli operaisti e dei teorici dell'impolitico, del pensiero negativo e della crisi (Cacciari, Esposito), il seme della 'differenza italiana', la sinisteritas di una tradizione politica e concettuale marginale, capace tuttavia di riconfigurare e rilanciare negli anni Novanta il paradigma biopolitico foucaultiano, proponendosi come alternativa alle esangue ripetizioni dei dispositivi concettuali della modernità.

I meriti del libro di Gentili sono molti, a partire dall'astuta decisione di utilizzare il dibatitto sull' 'Italian Theory' - che talvolta è poco più di una strategia pubblicistica per schivare un esercizio accademico di storia della filosofia e proporre invece una storia militante delle idee, un bilancio di ciò che è vivo e di ciò che è morto nell'attualità della lotta politica e dell'ecologia dei concetti.

A mio avviso, un libro cosi di parte in cui risuona tuttavia una voce così impersonale, una storia così lineare di una costellazione concettuale segmentata e minore, è di per sé un piccolo dono della nostra epoca, forse il frutto di una generazione che ha imparato a vivere senza Storia e senza Politica, e a resistere alle sirene della Filosofia, non per questo abbandonando la militanza e la gioia dei concetti.

'La filosofia ha bisogno, oggi più che mai, di categorie nuove, all'altezza dei tempi' (9). Poco importa dunque che il tragitto 'dall'operaismo alla biopolitica' narrato da Italian Theory non sia un destino dell'Essere ma un'efficace illusione prospettica, un'intersezione attualizzante di eventi e pensieri.

Gentili ci riferisce la consumazione, non tragica ma geologica, degli orizzonti politici occidentali e il misterioso emergere della vita come sfondo geo-filosofico - al contempo naturale e culturale, biologico e sociale, antropologico e storico - in cui sono immerse oggi le nostre parole e le nostre azioni.

Le pagine conclusive di Italian Theory sono dedicate al pensiero di Roberto Esposito e alla problematizzazione di che cosa sia 'una politica che ha origine nella vita' (221). Gentili non occulta la difficoltà di tematizzare e abitare antagonisticamente questo nuovo ambiente, chiudendo il volume con una lunga sequenza di domande senza risposte che riaprono il grande conflitto intorno allo 'stato di natura' occidentale. 


\section{Federico Luisetti}

University of North Carolina at Chapel Hill Dept of Romance Languages \& Literatures 123 Dey Hall, CB 3170

Chapel Hill, NC 27599 (USA)

luisetti@email.unc.edu 